\section{Effects of Contact Timing and Surface Roughness for Solid State Diffusion Bonding on Oxygen Free Copper}

\author{
Masaaki Tsukamoto*, Atsushi Yamamoto** \\ *Graduate Student, University of Hyogo \\ 2167 Shosha, Himeji, Hyogo, Japan \\ E-mail: ew10x002@steng.u-hyogo.ac.jp \\ **Division of Materials Science and Chemistry, Graduate School of Engineering, University of Hyogo \\ 2167 Shosha, Himeji, Hyogo, Japan
}

\begin{abstract}
Diffusion bonding on oxygen free copper showing a low flow stress at high temperatures was carried out with changing surface roughness and delaying contact between bonding surfaces until a temperature was raised up to bonding condition. Tensile strength was increased with decreasing the surface roughness, but a fracture occurred at the bonded interface even in the specimens not observed voids. While, they fractured at not the bonded interface but the base metal with delaying contact between bonding surfaces. It was attributed to removing oxide inclusions at the bonded interface. These oxide inclusions existed at the bonded interface of the specimens whose surfaces were chemically polished prior to the bonding.

Grain boundary migration across the bonded interface was generated only in the specimens given fine surface roughness and long bonding time. The behavior was scarcely depended on misorientation of grain boundaries. The fracture at the base metal was attained in the specimens retaining an original bonded interface. It would be indicated that joint strength was not affected by the grain boundary migration.
\end{abstract}

Key words: diffusion bonding, oxygen free copper, surface roughness, contact timing, grain boundary migration

\section{Introduction}

Oxygen free copper having high thermal conductivity has been largely applied as a material for cooling apparatuses. Diffusion bonding which can maintain high dimensional accuracy is adopted to construct a cooling circuit in the apparatuses. Oxygen free copper shows a significantly low flow stress at elevated temperatures. Diffusion bonding is preferable to be performed under low pressures to suppress deformation of works. However, the bonding is attained by applying optimum pressures which can eliminate voids at the bonded interface and disperse oxide layers on the bonding surface ${ }^{1)}$. Therefore, other factors for bonding mechanisms are required in order to obtain excellent joint properties under low bonding pressures.

It has been reported that surface roughness as well as temperature, pressure and time is one of the important factors affecting joint properties on diffusion bonding 2), 3). Irregularities on the bonding surfaces are hardly deformed in the case of low bonding pressures, so that the bonding surfaces cannot sufficiently adhere. Therefore, surface roughness would be closely related to joint properties on diffusion bonding under low 
pressures.

Oxide layers on the bonding surfaces interfere with progress for the bonding. Various surface treatments prior to the bonding such as chemical polishing have been proposed for removing the oxide layers. But they are formed again when the bonding surfaces are once exposed in an atmosphere after surface treatments. It has been reported that the oxide layers disappear by heating under an ultra high vacuum of $5.0 \times 10^{-6} \mathrm{~Pa}^{4)}$. The oxide layers can be decomposed under lower degree of vacuum in terms of temperature and oxygen partial pressure. Furthermore, the bonding surfaces are maintained as a free surface until a temperature is raised up to bonding condition, which would allow the oxide layers to promote decomposition.

In the present study, effects of surface roughness and contact timing for solid state diffusion bonding under low pressures on oxygen free copper were investigated.

\section{Experimental procedure}

Commercial oxygen free copper rods with 13 $\mathrm{mm}$ in diameter and $30 \mathrm{~mm}$ in length were used as a specimen for the present study. Bonding surfaces on the specimens were polished with an emery paper $\# 220$, \#1000, \#4000 and buffing followed by washing with acetone. Some of the specimens were chemically polished after mechanical polishing.

The prepared specimens were fixed at the edge of a upper carbon rod for loading to delay contact between bonding surfaces as shown in Fig.1. Diffusion bonding was carried out in a quartz tube below a pressure of $4.0 \times 10^{-3} \mathrm{~Pa}$. Bonding temperature, pressure and time were $1073 \mathrm{~K}, 2 \sim 4$ $\mathrm{MPa}$, and $3.6 \mathrm{ks}$ or $7.2 \mathrm{ks}$, respectively. Loading procedures were two patterns, in which contact between bonding surfaces followed by loading was performed before and after a temperature was raised up to bonding condition.

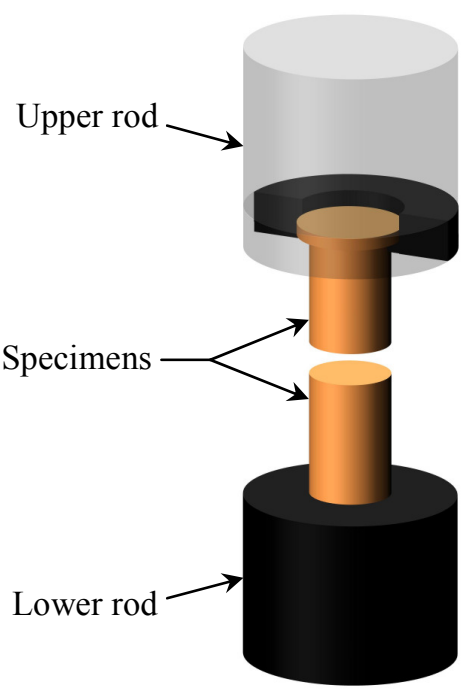

Fig.1 Schematic illustration for specimens setup.

The Bonded specimens were divided into two pieces. One pieces were turned to tensile specimens based on JIS and the mechanical properties were evaluated by a tensile test with a crosshead speed of $1.67 \times 10^{-2} \mathrm{~mm} / \mathrm{s}$. Fractured surfaces of the specimens were then observed with an SEM. The other pieces were cut perpendicular to the bonded interface and the cross sections were mechanically polished and electropolished. Microstructures near the bonded interface were then observed by means of an SEM-EBSD system in order to confirm grain boundary migration in detail.

\section{Results and discussion}

Bonded interfaces in the specimens fabricated at $1073 \mathrm{~K}$ for $3.6 \mathrm{ks}$ under bonding pressures varied from 2 to $4 \mathrm{MPa}$ are shown in Fig.2. Voids existed at the bonded interfaces in the specimens whose surfaces were polished with an emery paper \#220 and morphology of the voids was not also changed when the bonding pressure was increased. In the case of low bonding pressures, bonding surfaces are difficult to adhere by deforming the irregularities on the surfaces. Therefore, initial contact areas are important to obtain bonded areas, which is closely related to roughness on the bonding surfaces.

Bonded interfaces in the specimens whose surfaces were polished with emery papers 



Fig.2 Bonded interfaces in the specimens fabricated under a pressure of 2, 3 and $4 \mathrm{MPa}$, (a), (b) and (c), respectively. The bonding surfaces were polished with an emery paper \#220. The black arrows indicate the bonded interfaces.


Fig.3 Bonded interfaces in the specimens prepared by polishing the surfaces with emery papers \#1000, \#4000 and buffing, (a), (b) and (c), respectively. The black arrows indicate the bonded interfaces.

$\# 1000, \# 4000$ and buffing are shown in Fig.3. Bonding temperature, pressure and time were at $1073 \mathrm{~K}, 2 \mathrm{MPa}$ and for $3.6 \mathrm{ks}$, respectively. In the specimens prepared by polishing the surfaces with an emery paper \#1000, the number of voids at the bonded interface was increased, but the size of those was significantly decreased. No clear voids were observed at the bonded interface in the specimens prepared by polishing the surfaces with an emery paper \#4000 and buffing. Tensile strength of the specimens prepared by polishing the surfaces with an emery paper \#220 was approximately half as much as that of the base metal. The tensile strength was increased with decreasing the surface roughness and comparable to the base metal on the specimens prepared by polishing the surfaces with an emery paper \#4000 and buffing. Nevertheless, all of the specimens were fractured at the bonded interface.

Void shrinkage through interface/volume diffusion mechanisms requires quite a long time than that through plastic/creep deformation mechanisms ${ }^{5)}$ and is accelerated when voids are below about $2 \mu \mathrm{m}$ in size ${ }^{3)}$. The bonding surfaces need to be fine to obtain appropriate bonding areas under low bonding pressures. However, the fracture occurred at the bonded interface even in the specimens adequately gained an adhesion between bonding 

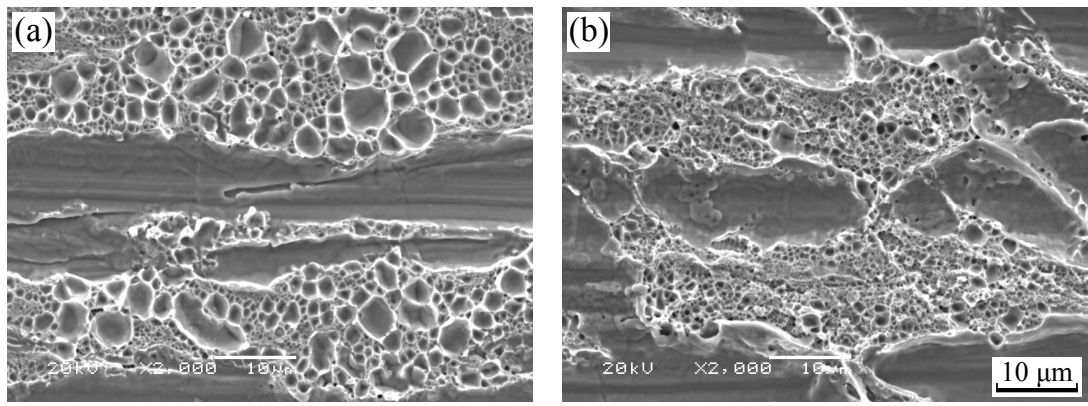

Fig.4 Fractured surfaces of the specimens bonded with and without contact between bonding surfaces until a temperature was raised up to bonding condition, (a) and (b), respectively.

surfaces.

Joint properties of the specimens were significantly improved by delaying contact between bonding surfaces until a temperature was raised up to bonding condition. The fracture occurred at not the bonded interface but the base metal in the specimens prepared by polishing the surfaces with an emery paper $\# 4000$ and buffing. Though the specimens prepared by polishing the surfaces with emery papers \#220 and \#1000 were all fractured at the bonded interface, the tensile strength was increased with the late contact procedure.

In the case of delaying contact between bonding surfaces, bonding time should be shorter than a conventional procedure in which bonding surfaces were contacted before a temperature was raised. Conversely, the joint properties were improved. The late contact procedure would work for inhibitors which deteriorate joint properties. Fractured surfaces of the specimens bonded with and without contact between bonding surfaces until a temperature was raised up to bonding condition are shown in Fig.4. Relatively large dimples were observed on the fractured surface in the specimens bonded by the conventional procedure, while they were not observed when the specimens were fabricated by the late contact procedure. The difference in size of dimples was not generated due to a strain rate because the bonding areas were almost equal each other. Large dimples would be resulted from inclusions at the bonded interface.

Oxide layers of copper can be decomposed at high temperatures in vacuum. The oxide

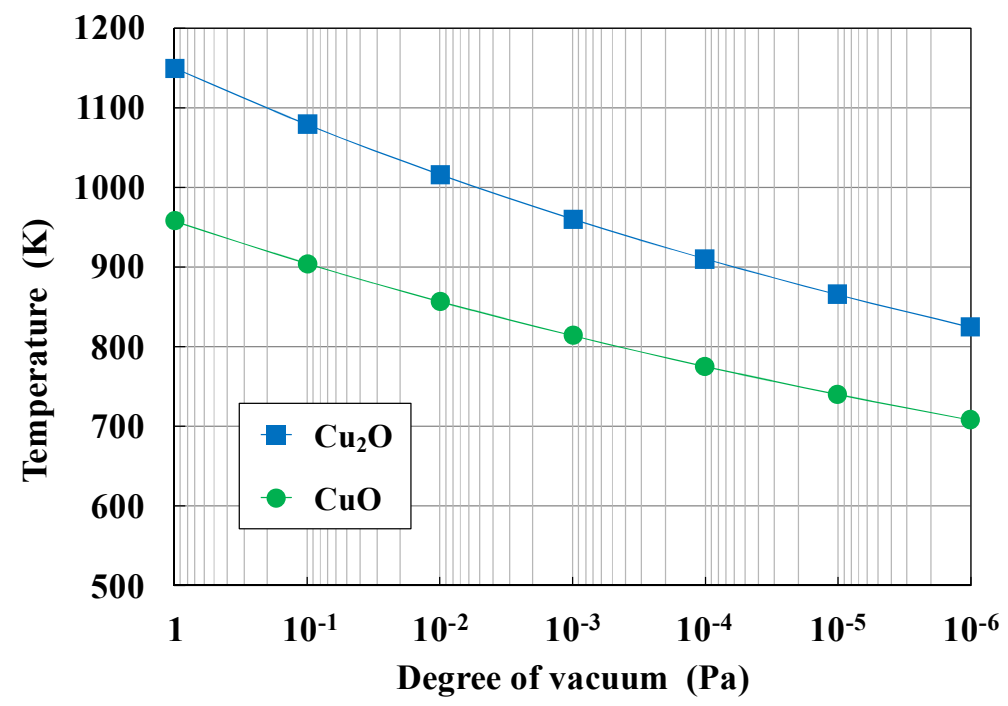

Fig.5 Relationships between temperature and degree of vacuum to decompose $\mathrm{Cu}_{2} \mathrm{O}$ and $\mathrm{CuO}$. 

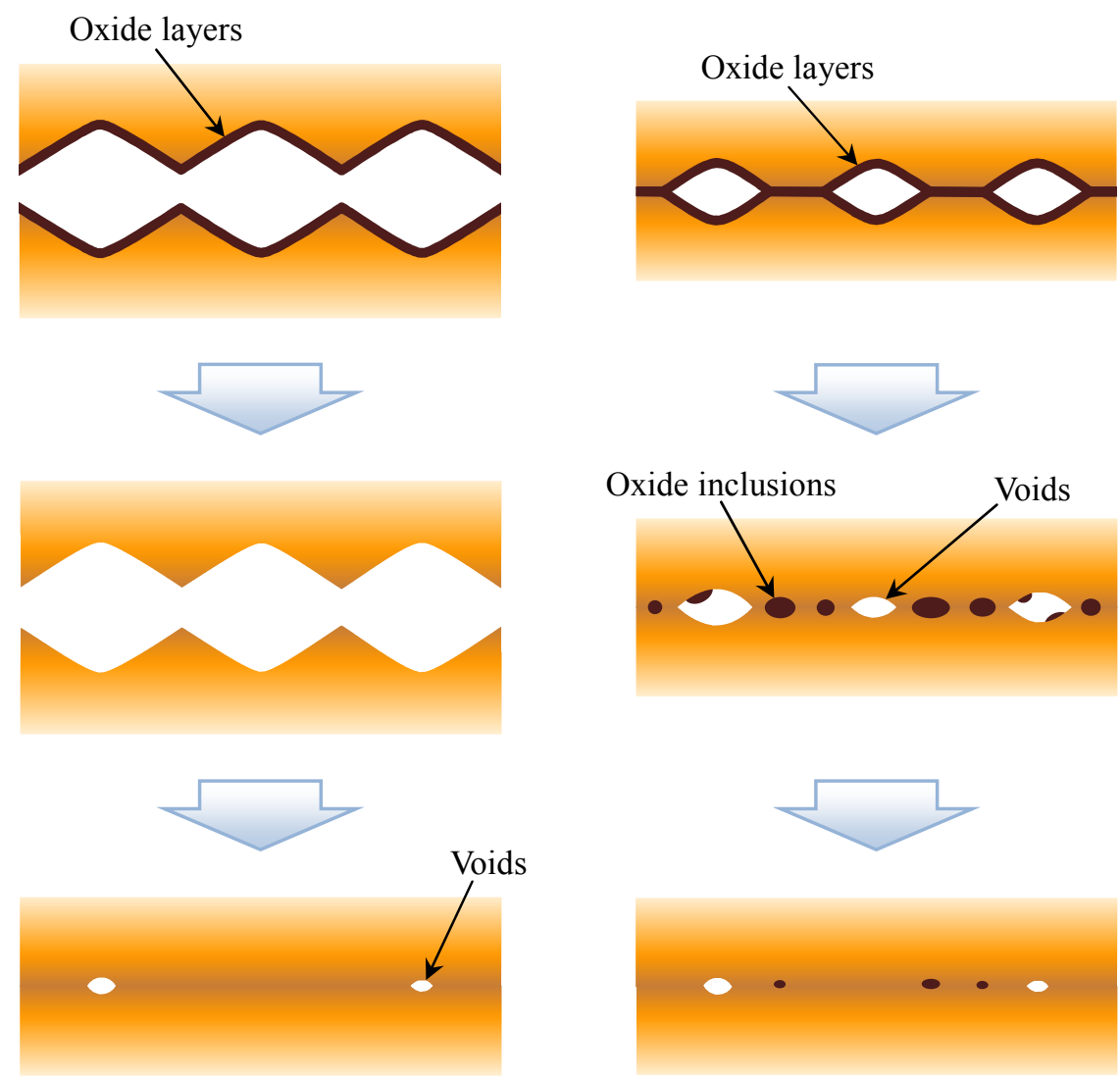

(a)

(b)

Fig.6 Schematic illustrations of changes at the bonded interfaces in the late contact and the conventional procedure, (a) and (b), respectively.

layers consist of $\mathrm{Cu}_{2} \mathrm{O}$ and $\mathrm{CuO}$, they can be decomposed when the standard free energy of formation $\Delta \mathrm{G}_{1}$ and $\Delta \mathrm{G}_{2}$ defined by equation (1) and (2), respectively, are negative values ${ }^{6}$.

$$
\begin{aligned}
& \Delta \mathrm{G}_{1}=40000-18.09 \mathrm{~T}+1.987 \mathrm{~T} \ln \mathrm{P}^{1 / 2} \quad \ldots \\
& \Delta \mathrm{G}_{2}=37290-22.24 \mathrm{~T}+1.987 \mathrm{~T} \ln \mathrm{P}^{1 / 2} \ldots
\end{aligned}
$$

where $\mathrm{T}$ is bonding temperature and $\mathrm{P}$ is oxygen partial pressure. Relationships between temperature and degree of vacuum calculated from these equations are shown in Fig.5. The oxygen partial pressure used was a value in the literature reported by other author ${ }^{7)}$. Decomposition of $\mathrm{Cu}_{2} \mathrm{O}$ and $\mathrm{CuO}$ progresses in the regions above each line. Both the oxides were decomposed during the bonding because the temperature and degree of vacuum in the present study were 1073 $\mathrm{K}$ and $4.0 \times 10^{-3} \mathrm{~Pa}$, respectively. These reactions occur in the case of bonding surfaces retained as a free surface as shown in Fig.6 (a). While, evacuation near the bonded interface was difficult in the case of the conventional procedure due to an adhesion between bonding surfaces, which disturbs the decomposition of the oxide layers. Oxide layers of copper gradually gather

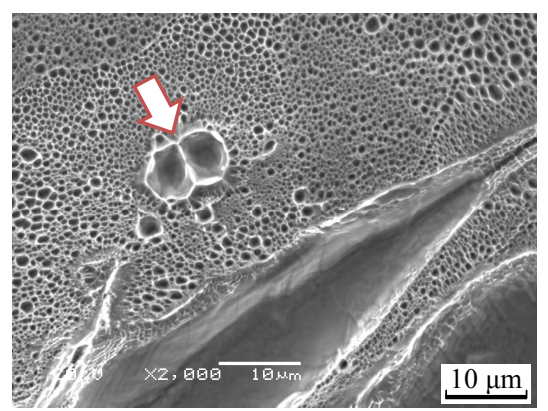

Fig.7 Fractured surface in the specimens whose surfaces were chemically polished after buffing. 



Fig.8 Microstructures near the bonded interfaces in the specimens prepared by polishing the surfaces with buffing. The bonding times were $3.6 \mathrm{ks}$ and $7.2 \mathrm{ks}$, (a) and (b), respectively. The black arrows indicate the bonded interfaces.

with bonding and result in formation of oxide inclusions as shown in Fig.6 (b). They are then reduced by diffusion of oxygen into the base metal. However, the oxides do not completely disappear at the bonded interface because solubility limit for oxygen in copper is extremely low. The residual oxides cause the fracture at the bonded interface.

The oxide layers are difficult to be removed by surface treatment prior to bonding such as chemical polishing. As shown by the arrow in Fig.7, relatively large dimples were observed in the fractured surfaces of the specimens chemically polished before bonding.
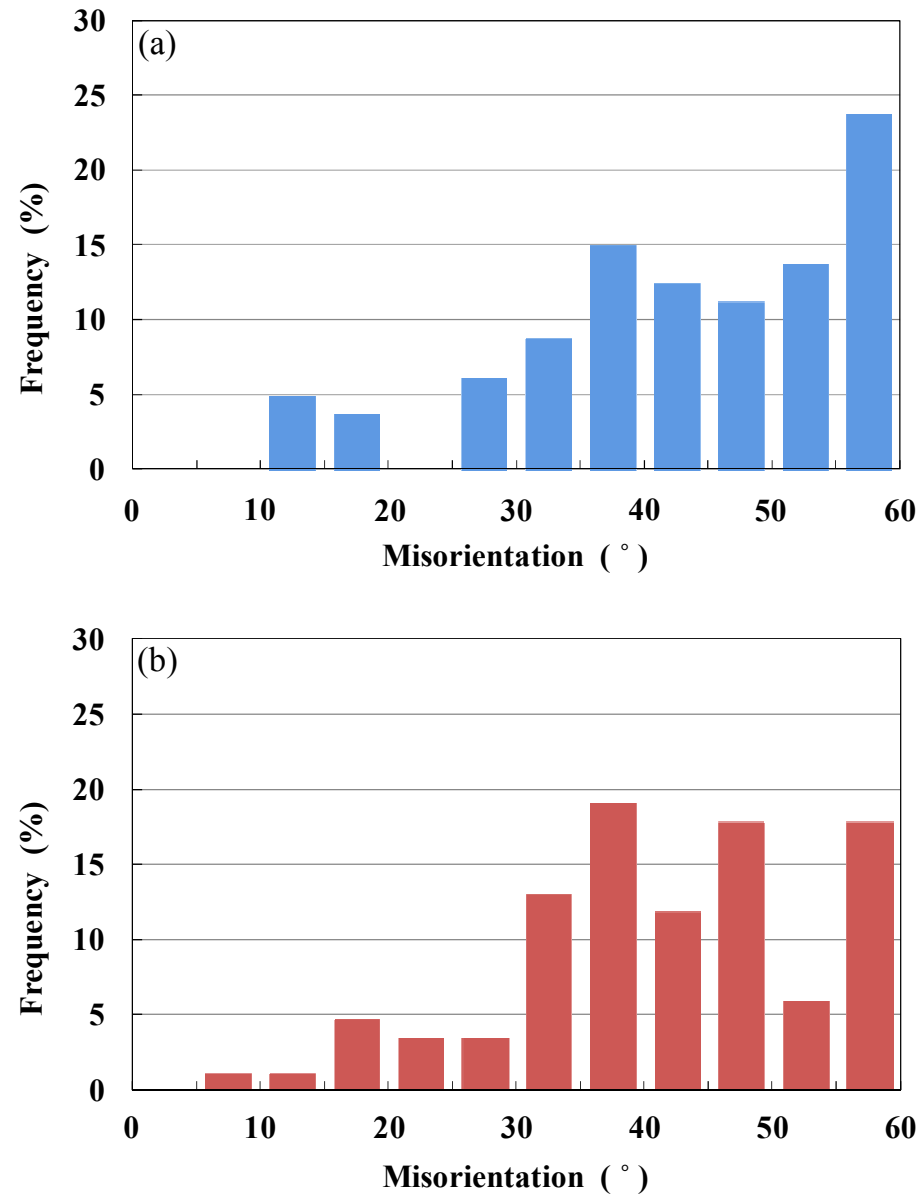

Fig.9 Distributions of misorientation on grain boundaries with and without migration across the bonded interface, (a) and (b), respectively. 
The oxides would remain at the bonded interface. Furthermore, difference in height among grains and grain boundaries were formed by chemical etching, which reduced bonded areas. The oxide layers are formed again on the bonding surfaces once the specimens are exposed in an atmosphere after chemical polishing. The oxide layers have to be removed in vacuum.

Grain boundary migration across the bonded interface remarkably occurred in the specimens polished with buffing when bonding time was prolonged up to $7.2 \mathrm{ks}$ regardless of contact timing as shown in Fig.8. The grain boundary migration would be due to a grain growth since recrystallization has already completed during raising a temperature. Furthermore, voids at the bonded interface play a role as a barrier to the grain boundary migration ${ }^{8}$. Therefore, the grain boundary migration requires a relatively long bonding time, which induces deformation of works. Distributions of misorientation on grain boundaries with and without migration across the bonded interface are shown in Fig.9. The distributions were calculated from about 100 grain boundaries for each measurement. Both the distributions indicated a similar tendency. The grain boundary migration was scarcely related to misorientation. In contrast, the specimens polished with an emery paper $\# 4000$ and buffing could fracture at the base metal even for $3.6 \mathrm{ks}$ in bonding time, which retained an original bonded interface. Therefore, joint strength would not be affected by the grain boundary migration.

\section{Conclusion}

Effects of surface roughness and contact timing for solid state diffusion bonding on oxygen free copper were investigated. Results in the present study were summarized as follows.

(1) Shrinkage of voids at the bonded interface was not progressed by deformation mechanisms under bonding pressures which enable the specimens to minimize deformation.

(2) Tensile strength was increased with decreasing roughness on the bonding surfaces. The specimens prepared by polishing the surfaces with an emery paper \#4000 and buffing showed the tensile strength comparable to the base metal, but a fracture nevertheless occurred at the bonded interface.

(3) These specimens fractured at not the bonded interface but the base metal with delaying contact between bonding surfaces until a temperature was raised up to bonding condition. Relatively large dimples were observed on the fractured surfaces of the specimens fabricated by a conventional procedure in comparison with those by the late contact procedure.

(4) Grain boundary migration across the bonded interface was significantly generated only in the specimens prepared with buffing and extended up to $7.2 \mathrm{ks}$ in bonding time irrespective of contact timing, and also the behavior was scarcely related to misorientation.

(5) A fracture at the base metal was attained even in the specimens maintaining the original bonded interface, which suggested that joint strength was not affected by grain boundary migration across the bonded interface.

\section{References}

1) K. A. Peterson, I. Dutta and M. Chen: J. Mater. Processing Technol. 145(2004) 99-108.

2) A. Wang, O. Ohashi, N. Yamaguchi, Y. Higashi, N. Hitomi and K. Takahashi: Trans. MRS-J 27(2002) 739-742.

3) O. Ohashi and T. Hashimoto: J. Japan Weld. Soc. 45(1976) 485-491.

4) O. Ohashi and Y. Asano: J. Japan Inst. Metals 61(1997) 744-749. 
5) K. Nishiguchi and Y. Takahashi: Quarterly J. Japan Weld. Soc. 3(1985) 303-309.

6) Y. Fukaya, Y. Higashi, T. Higo, M. Hamaoka, M. Sueda, T. Kobayashi, M. Ogawa and K. Shinozaki: Quarterly J. Japan Weld. Soc. 15(1997) 467-475.

7) O.Ohashi: Quarterly J. Japan Weld. Soc. 4(1986) 514-520.

8) T. Hashimoto and K. Tanuma: J. Japan Weld. Soc. 38(1969) 1225-1233. 